\title{
An Exploratory Study of IT-enabled Collaborative Process Modeling
}

\author{
Christopher Hahn ${ }^{1}$, Jan Recker ${ }^{2}$, Jan Mendling ${ }^{1}$ \\ ${ }^{1}$ Humboldt University, Unter den Linden 6, 10099 Berlin, Germany \\ ${ }^{2}$ Queensland University of Technology 126 Margaret Street, Brisbane QLD 4000, Australia \\ hanchris@cms.hu-berlin.de,j.recker@qut.edu.au,jan.mendling@wiwi.hu-berlin.de
}

\begin{abstract}
Process modeling is an important design practice in intra- as well as interorganizational process improvement projects. Inter-organizational process modeling often requires collaboration support for distributed participants. We present the results of a preliminary exploratory of study of process modeling on basis of collaborative technology. We examine a group of process modelers that rely on a collaborative modeling editor to complete two process modeling tasks in distributed settings. We examine how the participants learn to appropriate the technology, the key phases and tasks of collaborative process modeling, the breakdowns encountered and workarounds employed by the participants. With our study, we provide a first understanding of the IT-enabled process of process modeling, and detail a set of guidelines and implications for the research and design of collaborative process modeling.
\end{abstract}

Keywords: process modeling, collaboration, distributed modeling, collaborative technology.

\section{Introduction}

Information Technology has enhanced many work practices within large and small organizations. Specifically, the introduction of collaborative technology has provided organizational staff with the opportunity to engage in remote forms of collaboration, first by email, then via attachments in email, chatting, from text to multimedia forms involving audio and video, and, recently, to fully collaborative virtual environments [1].

Collaborative technologies have found widespread use by analysts in decision making [2], requirements engineering [3] and even complex design work [4]. Following this work, our interest in this paper is to examine how collaborative technology can be applied to process modeling - the design of graphical blueprints of inter- or intra-organizational business process for the act of process performance measurement, organizational re-design or workflow automation.

Process modeling is typically performed using process modeling grammars [5], semi-formal notations that provide graphical elements to map out business processes in terms of the tasks that have to be performed, the actors that are involved in the execution of these tasks, relevant data and sources (papers, forms, systems and technology) of the data, and the business rule logic that describes the logical and temporal order in which tasks are to be performed [5]. While a variety of tools are available to create and analyze these models of business processes, studies and anecdotal evidence alike still report challenges in the process of process modeling, 
most notably in the phases of eliciting business process information from relevant stakeholders, and formalizing them in process model [6]. Some authors have argued that this challenge is due to a lack of support for the process of process modeling, i.e., support for the collaboration between business analysts and domain experts in the development of process models [7].

This challenge is exacerbated further in globalized setups of organizations and projects in which cross-organizational processes need to be designed. This is because in these contexts, required modeling stakeholders (e.g., analysts, project managers and domain experts) are often geographically dispersed and need to engage in the process modeling effort from remote locations. Yet, while such remote collaborative process modeling could, theoretically, benefit from collaborative technology as in use in other organizational tasks (e.g., project management [8]), to date, tool solutions have only recently begun to explore potential support features for collaborative process modeling [e.g., 9]. Still, the effect the emerging collaborative technology has on the process or outcome of process modeling is yet to be understood.

In this paper, therefore, we examine collaborative process modeling using a novel research prototype for collaborative process modeling on basis of the Google Wave technology (http://wave.google.com). Specifically, we examine in two settings how a group of process modelers working in a distributed setting are appropriating the collaborative process modeling technology, and how the process of collaborative process modeling is carried out.

The sections that follow first describe related work in the areas of process modeling, collaboration and collaborative technology. We then discuss the setup of our empirical study and how we collected and analyzed data. In section 4 we discuss the findings from our exploratory study. We discuss the emerging implications for research and technology design. Then, we conclude the paper with a review of contributions, limitations and an outlook to future work.

\section{Related Work}

\subsection{The Process of Process Modeling}

To be able to gauge the potential positive and negative consequences of collaborative technology on process modeling in distributed environments, an understanding of the process and product of process modeling is required first. In the context of this article we understand process modeling as an act of facilitating a shared understanding and to increase knowledge about a business process domain [10].

Several studies have examined the process of modeling in terms of its key phases or stages [7] and the main involved roles [11]. Specifically, it was found that process modeling often is not conducted in a linear fashion but is a rather repetitive and cyclic task that is going back and forth, thereby re-defining the problem, re-setting the borders or re-iterating and revising the artifact. Rittgen [12] has proposed a set of negotiation models to understand the patterns of human interactions in the process of 
process modeling. He [13] also studied differences in the final product (i.e., the model/ script) with collaborative tool support. Ssebuggwawo et al. [14] examined collaborative modeling by looking for rules, goals and interactions proposing the notion of Modeling Games. All of the above work used setups where participants were locally attendant and therefore be able to communicate directly.

We argue that the characteristics of collaboration (distributed participants around the globe) pose different requirements to the modeling process and its tool support. Furthermore the findings of recently mentioned work do not give implications for collaboration technology design.

\subsection{Collaboration and Collaborative Technology}

Collaboration and collaborative technology has already been applied and examined in related areas such as design or learning. For example, Susman et al. [15] synthesized and extended existing theories on the appropriation of collaborative technologies in new product development by "recognizing misalignments between technology, task, organization and the group." Marjanovic [16] validated an interactive methodology for learning and teaching in a synchronous electronic collaborative environment emphasizing the necessity of understanding collaborative processes in order to design better methodologies.

Our interest in this study is to look how collaborative technology can be used for process modeling and what effects are observable from this technology appropriation. We selected the research prototype Gravity as a possible collaboration technology. Gravity is a collaborative modeling editor on the basis of Google Wave. It enables to communicate (chatting) and to model in real time thereby largely supporting BPMN in its version 1.0, albeit some grammar constructs are missing, such as pools and swim lanes. Furthermore, it automatically keeps a history log that can be viewed later again and therefore supports data collection and analysis in a research setting.

\section{Research Design}

\subsection{Study Overview}

We decided not to offer a priori hypotheses and instead opted for an exploratory study design. Consequently, our objective was to observe the practices employed in the collaborative modeling environment without having expectations about the efficacy of such a setup (e.g., in terms of accuracy, efficiency or other criteria).

To be able to collect sufficient data whilst maintaining control over potentially confounding external factors, we selected a quasi-experimental design [17].

Our explorative study is structured with the help of the framework for evaluating conceptual modeling techniques (CMT) proposed by Gemino and Wand [18]. This framework is based on two main dimensions. The first dimension comprises factors 
that affect the conceptual modeling technique whereas the second consists of affected factors (i.e. outcomes).

The first affecting factor is content to be delivered, which refers to the type of information contained within the cases. We selected two cases to be modeled each describing a different domain, but both being described in a process-oriented way. The participants did not receive the complete description but instead only partial information, which each represented the knowledge of a specific role has in the process (domain experts, e.g., a clerk, an administrative office, a line manager). For example, our second case described a purchase order process in a company and the partial information given to one participant refers to the role of the requester. In total, all relevant information was given to the participants but it required them to communicate in order to assemble the case.

The second affecting factor, presentation of content, includes the following dimensions:

- The choice of grammar constructs to consider: We include all BPMN constructs that are available in the selected collaborative modeling technology (SAP Gravity).

- The nature of comparison (within or between grammars): Since this study does not aim to compare different grammars it denotes an intra-grammar comparison (always BPMN) [19].

- Rules regarding the use of the grammar and how it is applied: We do not change the grammar rules, instead all BPMN rules should be applied (if possible).

- The way the script is presented (text, graphics, narrated, animated, etc.): Each participant was distributed using Wave \& Gravity for Collaboration support which offers textual representation (Google Wave) as well as graphical features (SAP Gravity).

The third affecting dimension describes the characteristics of the persons participating in the communication. Gemino and Wand [18] suggest to classify the participants' experience with respect to conceptual modeling and domain knowledge. Our participants varied alongside both dimensions to be able to examine effects stemming from different levels of domain or modeling knowledge. Still, we did not capture explicitly the experience levels of the participants in this study, but we will in our overarching research project.

The last dimension to be considered is the task itself, which usually is either model creation ('writing') or model viewing ('interpretation'). In our research we will focus on the act of model creation, thereby complementing the active stream of research investigating process model interpretation [e.g., 20].

The dimension of affected variables comprises observable outcomes of the tasks that can be used as the source of dependent measures in empirical comparisons. Focus of observation points to what has been measured, either the process or the product of using a CMT. Our interest is on the process of process modeling. The criterion for comparison refers to the measurement criteria that have been applied. These criteria can either be effectiveness or efficiency focused in nature [18]. The criteria we choose are focused on understanding the process of creating the model, both in terms of effectiveness and efficiency of the modeling process. Our measurements include the formation of requirements specification and elicitation actions performed, observable 
modeling actions, modeling phases and milestones, as well as the number and type of communication breakdowns across these stages.

In summation, our study design provides an opportunity to obtain a deeper understanding of the process of collaborative process modeling, and the actions taken within. This understanding, in turn, can lead to design guidelines for the development of technology-based support instruments to support process modeling, as well as the development of normative guidelines (checklists, instructions etc.) for the conduct of process modeling projects in collaborative settings. Of course, due to the limitations of this research setup in terms of ecological validity, our study will also set the stage for a large study using repeated field setting.

\subsection{Setup}

The four selected participants are Business Process Management researchers, each with a slightly different background. Specifically, there are two $\mathrm{PhD}$ students in information systems with extensive experience in process modeling, one $\mathrm{PhD}$ student in computer science with less experience in process modeling and one senior researcher with background in conceptual modeling but not process modeling. Participation was voluntary, with the only incentive offered being free food during the experiment. The workplaces were distributed across several research offices. Each participant was assigned an individual workstation.

The instructions are constrained to the goal of collaboratively modeling the described cases using only Gravity for communication. The objective of the modeling was to collaboratively produce an accurate and understandable BPMN process of the given process.

\subsection{Materials}

A case description (provided in disassembled form to the participants) was sent out ten minutes before the experiment officially started via e-mail. Case 1 described a mail distribution process, the description of which we separated according to the involved roles (mail processing unit, registry and cashier). The second case described a procurement process, again separated according the involved roles (requester, approver, purchase department clerk, clerk at goods receipt department and financial department clerk).

During the modeling sessions, participants were monitored to enforce silence (i.e., no active verbal communication) throughout the study, to simulate a geographically distributed setup. The modeling tool provided chatting but not VoIP functionality albeit, admittedly, such functionality could have been used through other software (e.g., Skype). Participants were given two hours in total to solve both cases. Due to technical difficulties with the tool prototype, both cases could not be modeled completely. This breakdown brings forward a limitation pertaining to the scope of study (e.g., we could not collect data on model validation activities conducted), but otherwise did not affect the data collection. After the second case, a focus group with the research team and the participants was conducted, to gather data about 
impressions, feedback and thoughts. The focus group meeting was audio-recorded, and notes were taken during the session.

\subsection{Measurement}

In order to measure the effectiveness of the modeling process we use the notion of breakdowns [21], which observed difficulties that occur during problem-solving processes [22]. We apply this notion to the process of process modeling using a categorization based on three steps of the semiotic ladder [23], following Rittgen's [12] classification of modeling as a language act on basis of Stamper's [23] theory of signs. Although Stamper [23] proposed four ladder steps, we will constrain our categorization on the originally used stages in semiotics [24], viz., syntax, semantics and pragmatics.

Specifically, we will summarize breakdowns related to the organization of the modeling session (e.g. setting the agenda), tool features requests (e.g. video conferencing) and tool deficiencies (e.g. errors) as pragmatic breakdowns. The semantic level will comprise breakdowns associated to the elicitation of the problem domain (e.g. defining the sequence) and mapping the domain to notation constructs (e.g. classifying activities). Impediments in applying the grammar of the notation are classified as syntactic breakdowns (e.g. restrictions for the usage of sequence flows in BPMN).

\section{Results and Discussion}

\subsection{Findings}

\section{Process of Modeling - Case Comparison}

In this section we will present similarities and differences found at comparing the process of process modeling employed by the participants for each case. For visualization purposes, we adapted BPMN diagrams to conceptualize these processes. In particular, we abstracted observable activities (BPMN symbol: activity) and status notifications (BPMN symbol: Signal Event). Furthermore, all breakdowns identified in the process are depicted as error event symbols. Annotations are provided to capture evidence from the observed actions and communications. The applied color codification scheme on activities indicates their occurrence in both cases as follows:

- white - found in both cases and at the same position

- grey - found in both cases but different position

- black - uniquely found in one case

Figure 1 depicts the process of modeling for the first case. In the beginning the participants agreed on the task and pasted the given case information pieces to make them available to all. We noticed that, during this case data collection phase, someone directly started to model. Subsequently the participants agreed to paste all case 
descriptions and defined their interdependencies as well as the respective sequence. Then they started modeling, concurrently the session suffered from several breakdowns. The agenda had to be revised, as one participant noticed a missing part and offered to model this part of the case. Additionally, lessons learnt were raised as they noticed issues ("We should have discussed the modeling approach first"). The participants were not able to complete the model because of a steadily reoccurring error of the modeling prototype. Therefore no final verification or validation activities could be observed. Still, we observed "on the fly" attempts to verify and validate the model, e.g., to connect two modeled parts ("Sort Mail should be connected to Register Mail"). In total 9 breakdowns (7 pragmatic, 1 semantic, 1 syntactic) were observed during case 1 .

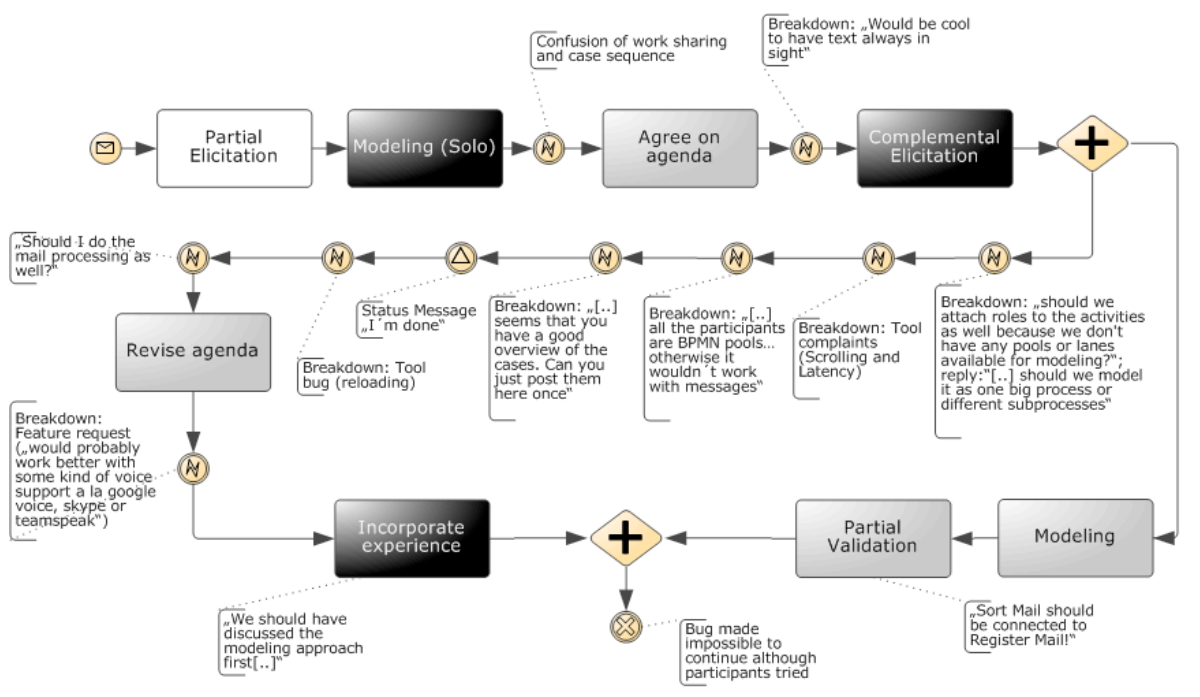

Figure 1. Process of Modeling for Case 1.

Figure 2 depicts the process of modeling for the second case. Similar to case 1 the participants started with a partial elicitation which in this situation means they pasted all relevant case information but did not agree on their sequence. In contrast to the first case, the participants structured the modeling session by agreeing on individually modeling exactly the role each participant received, which points to a learning effect carried over from the first case.

During this process, the participants discovered problems due to insufficient modeling space, which, in turn caused interferences to the modeling process and forced them to adapt the agenda. This breakdown became more cumbersome as the size of the "individual" models rose.

Similar to case one, the given process scenario could not be modeled completely due to re-occurring prototype functionality issues. Hence, we were not able to observe final verification or validation activities or discussions although, again, interactions indicated a partial validation (e.g. missing connection of the "individual" models: "someone has to consolidate the models"). In case 2 we found 3 breakdowns that are all on the pragmatic level. 


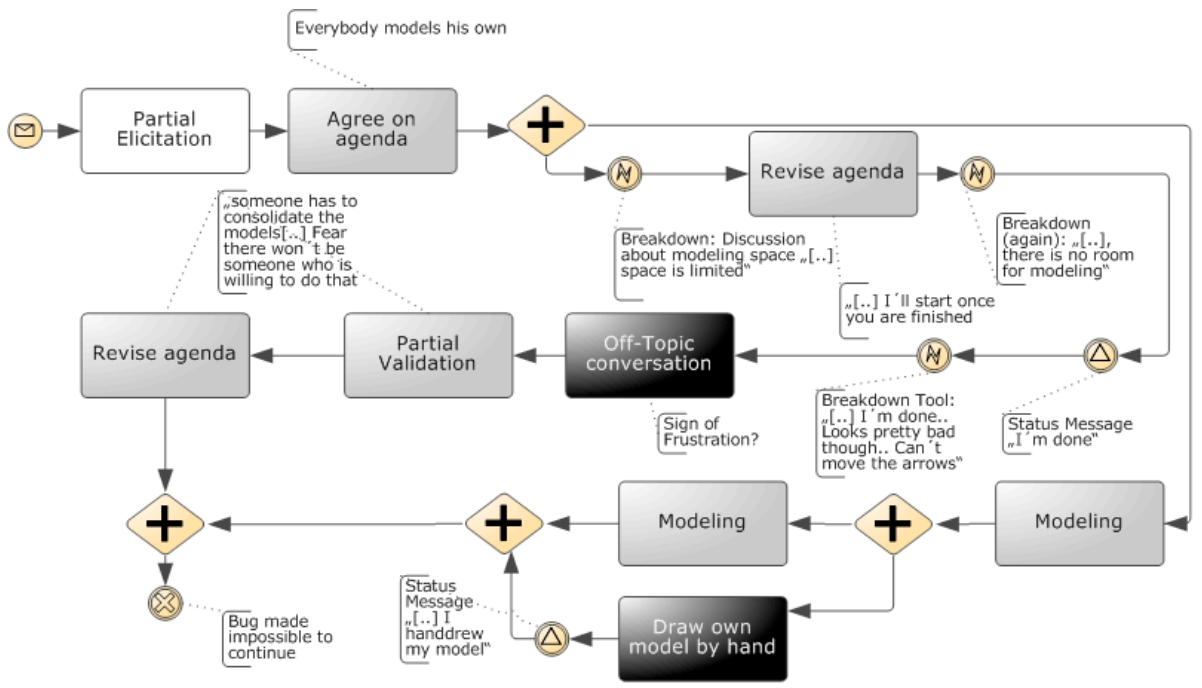

Figure 2. Process of Modeling for Case 2.

Comparing both cases we found one activity at the same position in both cases (partial elicitation), and a further four activities (agree on agenda, modeling, revise agenda, partial validation) that occurred in both cases albeit in a different order. This finding would suggest that these activities appear essential (but non-exhaustive) for a collaborative modeling session. The activity 'complementary elicitation' was only observed in case 1 , as in case 2 no sequence has been defined. A possible reason is the chosen modeling approach in case 2 that did not require a sequence definition upfront. Therefore, we would expect this activity to be done at the end of the session if it has not been interrupted. Similarly, the same holds for a final validation. The immediate individual modeling activity observed at the beginning of case 1 could possibly be ascribed to his/her curiosity of how the tool works. The "incorporate experience" activity in case 1 could reflect a familiarization effect with the new environment (group, tool, collaboration etc.). Both residual activities ("off-topic conversation" and "draw own model by hand") in case 2 are considered as effects (i.e. frustration and workaround) of a breakdown.

The comparison of the number of breakdowns in each process shows that there are a lot more breakdowns during the first case (case 1: 9, case 2: 3). Possible explanations are learning effects associated with the attempt of technology appropriation. Furthermore, due to the subsequent accomplishment of both cases participants may have regarded mentioning of similar problems as needless. The elaboration and categorization of breakdowns will follow in the next section.

\section{Breakdowns}

We now discuss the number and types of breakdowns identified during both modeling cases. We group the observed breakdowns according to the categorization proposed in section three and provide quote examples in order to show evidence. In total, we 
classified the 12 observed breakdowns into 5 breakdowns types on a pragmatic $(\mathrm{P}), 1$ on a semantic (SE) and 1 on a syntactic (SY) level.

- P.1 Information Elicitation: information representation (i.e., case description to all participants) was requested to be visible at all times ("Would be cool to have text always in sight", "[..] can you just post them here once"; observed in case 1)

- P.2 Organization/ Agenda Setting: participants did not know what to do (next) or how to share work ("We should have discussed modeling approach first", "Should I copy my text?", "Should I do the mail processing as well?"; observed in case 1)

- P.3 Tool Feature Request: support for a more natural communication was requested as a result of dissatisfaction ("[..] would probably work better with some kind of voice support[..]"; focus group interview; observed in case 1)

- P.4 Tool Deficiency: handling issues prevented participants from what they wanted to do (e.g. scrolling), Latency, Errors (e.g. steady reloading) ("[..] I can't scroll[..]", "[..] system keeps reload all the time"; observed in both cases)

- P.5 Tool Feature Request: insufficient modeling space resulted in conflicts at concurrent modeling ("[..] there is no room for modeling"; observed in case 2)

- SE.1 Process Decomposition: participants struggled in structuring the process model appropriately beforehand ("should we model it as one big process or different sub-processes based on different roles?"; observed in case 1)

- SY.1 Construct Grammar Rules: participants needed to recapitulate grammar rules as an effect of missing constructs ("[..] all participants are BPMN pools.. otherwise it wouldn't work with messages"; observed in case 1)

It was clearly observable that the participants tried to better organize the session in case 2 (how to proceed and who does what). A re-organization became necessary through additionally discovered tool limitations. Other breakdowns have not occurred or not been articulated again. Especially on the syntactic and semantic level, this might be either because it has been already cleared in the case 1 or the process description for case 2 does not demand for this thinking.

The dominance of breakdowns on the pragmatic level poses an important question: Does collaboration technology supporting process modeling require more features for organizational guidance? Due to the fact that both cases had to be aborted and we included tool issues in this category, we will neither support nor decline this hypothesis but instead note it as an interesting avenue for future study.

\subsection{Discussion}

In the last section we learned about activities and breakdowns occurring in collaboration technology supported process modeling. In our exploratory study, we observed two main findings. First, we identified similar activities present in both cases and derived possible explanations for differences. Second, we found that participants learned to appropriate the working environment provided by the technology, and adapted the modeling process such that the modeling could be carried out within the framework of conditions set by the tool. 


\section{Implications for Future Research}

Our findings have important implications for future research on process modeling.

Notably, we observed not only breakdowns but also breakdown workarounds employed by the participants in the collaborative modeling process. These actions indicate a familiarization process by the participants during their continued technology use. Future studies should study how this familiarization process is enacted in the context of collaborative modeling, and how it affects both the process and product of modeling.

Secondly, in our study we examined the process of process modeling on the basis of collaborative technology. A complementary stream of research could build upon our conceptualization of the IT-enabled modeling process to study the emergent implications on the outcome of the modeling process, i.e., the quality of the model produced. Such work could build on existing work on different quality measures of process models, e.g., soundness [25], understandability [20] or re-use [26].

\section{Implications for Technology Design}

Our findings further provide information for the design of collaborative technology. Specifically, we believe that the breakdowns we identified can be used to inform a design agenda to (re-) develop or address features that can assist to prevent or overcome those breakdowns.

P.1. (Information Elicitation) suggests the importance of a feature that allows the textual description of the domain to be displayed throughout the modeling activities.

P.2. (Organization/ Agenda Setting) was mainly overcome with the help of chat. While an initial discussion needs to be done to agree on the agenda, other mechanisms seem more appropriate to guide und support participants (e.g. keep track of work packages, their status as well as completion notifications). The request for organizational guiding is supported by the observed status messages during both cases and the focus group.

P.3. (Tool Feature Request (Natural Communication)) addresses the need for faster communication. In both cases, instant messaging functionality was provided as the only communication feature. Prominent weaknesses are the slow communication pace and "complexity" of several ways to reply in Google Wave which was communicated during the experiment and the focus group. Nevertheless, the chat (especially the collaborative messaging) can be used to support communication. A possible scenario would be to take notes during brainstorming or elicitation that are visible to all participants and afterwards provide these into the Dialogue Document within the tool.

P.4. (Tool Deficiencies) need to be addressed by the tool vendor as they lead to frustration among participants.

P.5. (Tool Feature Request (Modeling Space)) requires a greater modeling panel to avoid conflicts in modeling activities. The possibility to collaboratively edit a model on the same panel seemed to create a greater visibility which is supported by on the fly validation and verification activities in both cases.

SE.1. (Process Decomposition) suggests for specific modeling guidelines or rules that help the participants to structure their model. A possible implementation can be performed through, for instance, a checklist or FAQ using emerging knowledge on process model understandability [20]. 
SY.1. (Construct Grammar Rules) suggests for a syntactical check during the modeling. Although the research prototype of Gravity does not offer this feature, there are several other (single) modeling environments available providing such functionality.

In summary, we proposed requirements for collaborative support in process modeling addressing various aspects. In particular, a technology must provide support to modeling (P.1., P.5., SE.1., SY.1.), communication (P.3.) and coordination (P.2.).

\section{Conclusion}

In this paper we reported on an exploratory study of distributed process modeling with collaborative technology. Through a quasi-experimental setup we obtained preliminary insights about activities and breakdowns in collaborative process modeling, and identified a number of technology features that support various stages of the collaborative modeling exercise.

Our research bears some limitations. Specifically, this paper reports on an exploratory examination of 4 people modeling 2 cases. Therefore, we are well aware that our study is preliminary in nature. Still, we believe that our initial findings already inform an emerging body of knowledge and will also be useful to use in our work that follows. In moving forward from our study, we aim to replicate our experimental studies with larger cohorts of users. In doing so, we will incorporate the experiences gathered in this study resulting in adjusted setup.

\section{References}

1. Benford, S., Greenhalgh, C., Rodden, T., Pycock, J.: Collaborative Virtual Environments. Communications of the ACM 44 (2001) 79-85

2. Kiesler, S., Sproull, L.S.: Group Decision Making and Communication Technology. Organizational Behavior and Human Decision Processes 52 (1992) 96-123

3. Brouse, P.L., Fields, N.A., Palmer, J.D.: A Multimedia Computer Supported Cooperative Work Environment for. Requirements Engineering. In: Proceedings of the International Conference on Systems, Man and Cybernetics. IEEE, Chicago, Illinois (1992) 954-959

4. Davis, A., Murphy, J., Owens, D., Khazanchi, D., Zigurs, I.: Avatars, People, and Virtual Worlds: Foundations for Research in Metaverses. Journal of the Association for Information Systems 10 (2009) 90-117

5. Recker, J., Rosemann, M., Indulska, M., Green, P.: Business Process Modeling: A Comparative Analysis. Journal of the Association for Information Systems 10 (2009) 333363

6. Koschmider, A., Song, M., Reijers, H.A.: Social Software for Business Process Modeling. Journal of Information Technology 25 (2010) In Press

7. Frederiks, P.J.M., van der Weide, T.P.: Information Modeling: The Process and the Required Competencies of Its Participants. Data \& Knowledge Engineering 58 (2006) 4-20

8. Lee-Kelly, L.: Locus of Control and Attitudes to Working in Virtual Teams. International Journal of Project Management 24 (2006) 234-243 
9. Brown, R., Recker, J.: Improving the Traversal of Large Hierarchical Process Repositories. In: Schepers, H., Davern, M.J. (eds.): Proceedings of the 20th Australasian Conference on Information Systems. Association for Information Systems, Melbourne, Australia (2009) $144-153$

10. Recker, J.: Continued Use of Process Modeling Grammars: The Impact of Individual Difference Factors. European Journal of Information Systems 19 (2010) 76-92

11. Stirna, J., Persson, A., Sandkuhl, K.: Participative Enterprise Modeling: Experiences and Recommendations. In: Krogstie, J., Opdahl, A.L., Sindre, G. (eds.): Advanced Information Systems Engineering - CAiSE 2007. Lecture Notes in Computer Science, Vol. 4495. Springer, Trondheim, Norway (2007) 546-560

12. Rittgen, P.: Negotiating Models. In: Krogstie, J., Opdahl, A.L., Sindre, G. (eds.): Advanced Information Systems Engineering - CAiSE 2007. Lecture Notes in Computer Science, Vol. 4495. Springer, Trondheim, Norway (2007) 561-573

13. Rittgen, P.: Collaborative Modeling - A Design Science Approach. In: Proceedings of the 42nd Hawaii International Conference on System Sciences. IEEE, Waikoloa, Big Island (2009) $1-10$

14. Ssebuggwawo, D., Hoppenbrouwers, S.J.B.A., Proper, E.: Interactions, Goals and Rules in a Collaborative Modelling Session. In: Persson, A., Stirna, J. (eds.): The Practice of Enterprise Modeling - PoEM 2009. Lecture Notes in Business Information Processing, Vol. 39. Springer, Stockholm, Sweden (2009) 54-68

15. Susman, G.I., Gray, B.L., Perry, J., Blair, C.E.: Recognition and Reconciliation of Differences in Interpretation of Misalignments When Collaborative Technologies Are Introduced into New Product Development. Journal of Engineering and Technology Management 20 (2003) 141-159

16. Marjanovic, O.: Learning and Teaching in a Synchronous Collaborative Environment. Journal of Computer Assisted Learning 15 (1999) 129-138

17. Cook, T.D., Campbell, D.T.: Quasi-Experimentation: Design and Analysis Issues. Houghton Mifflin, Boston, Massachusetts (1979)

18. Gemino, A., Wand, Y.: A Framework for Empirical Evaluation of Conceptual Modeling Techniques. Requirements Engineering 9 (2004) 248-260

19. Gemino, A., Wand, Y.: Evaluating Modeling Techniques based on Models of Learning. Communications of the ACM 46 (2003) 79-84

20. Mendling, J., Reijers, H., van der Aalst, W.M.P.: Seven Process Modeling Guidelines (7PMG). Information and Software Technology 52 (2010) 127-136

21. Guindon, R., Krasner, H., Curtis, B.: Breakdowns and Processes During the Early Activities of Software Design by Professionals. In: OIson, G.M., Sheppard, S., Soloway, E. (eds.): Empirical Studies of Programmers: Second Workshop. Ablex Publishing, Norwood, New Jersey (1987) 65-82

22. Vessey, I., Conger, S.A.: Requirements Specification: Learning Object, Process, and Data Methodologies. Communications of the ACM 37 (1994) 102-113

23. Stamper, R.K.: The Semiotic Framework for Information Systems Research. In: Nissen, H.E., Klein, H.K., Hirschheim, R. (eds.): Information Systems Research: Contemporary Approaches and Emergent Traditions. North Holland, Amsterdam, The Netherlands (1991) 515-528

24. Morris, C.W.: Writings on the General Theory of Signs. Mouton de Gruyter, The Hague, The Netherlands (1971)

25. Verbeek, H.M.V., van der Aalst, W.M.P., ter Hofstede, A.H.M.: Verifying Workflows with Cancellation Regions and OR-joins: An Approach Based on Relaxed Soundness and Invariants. The Computer Journal 50 (2007) 294-314

26. Irwin, G.: The Role of Similarity in the Reuse of Object-Oriented Analysis Models. Journal of Management Information Systems 19 (2002) 219-248 